\title{
Feasibility study to objectively assess activity and location of Hispanic preschoolers: a short communication
}

\author{
Teresia M. O’Connor ${ }^{1,2}$, Ester Cerin ${ }^{3}$, Jessica Robles ${ }^{1}$, Rebecca E. Lee ${ }^{4}$, Jacqueline Kerr ${ }^{5}$, \\ Nancy Butte ${ }^{1}$, Jason A. Mendoza ${ }^{1,2}$, Deborah Thompson ${ }^{1}$, Tom Baranowski ${ }^{1}$ \\ ${ }^{1}$ USDA/ARS Children's Nutrition Research Center, Pediatrics, Baylor College of Medicine, Houston, TX 77030 \\ USA; ${ }^{2}$ Academic General Pediatrics, Pediatrics, Baylor College of Medicine, Houston, TX 77030 USA; \\ ${ }^{3}$ Institute of Human Performance, University of Hong Kong, Hong Kong; ${ }^{4}$ Texas Obesity Research Center, \\ Health and Human Performance, University of Houston, Houston, TX 77204, USA; ${ }^{5}$ Department of Family \\ and Preventive Medicine, University of California, La Jolla, CA 92093, USA
}

\begin{abstract}
Both physical and social environmental factors influence young children's physical activity, yet little is known about where Hispanic children are more likely to be active. We assessed the feasibility of simultaneously measuring, then processing objective measures of location and physical activity among Hispanic preschool children. Preschool-aged Hispanic children $(n=15)$ simultaneously wore QStarz BT100X global positioning system (GPS) data loggers and Actigraph GT3X accelerometers for a 24- to 36-hour period, during which time their parents completed a location and travel diary. Data were aggregated to the minute and processed using the personal activity location measurement system (PALMS). Children successfully wore the GPS data loggers and accelerometers simultaneously, 12 of which yielded data that met quality standards. The average percent correspondence between GPS- and diary-based estimates of types of location was high and Kappa statistics were moderate to excellent, ranging from 0.49-0.99. The between-method (GPS monitor, parent-reported diary) correlations of estimated participant-aggregated minutes spent on vehicle-based trips were strong. The simultaneous use of GPS and accelerometers to assess Hispanic preschool children's location and physical activity is feasible. This methodology has the potential to provide more precise findings to inform environmental interventions and policy changes to promote physical activity among Hispanic preschool children.
\end{abstract}

Keywords: preschool-aged children, physical activity, global positioning system, accelerometer, Hispanic.

\section{Background}

Previous studies suggest that Hispanic-American preschoolers may be less physically active than nonHispanic White preschool-aged children (Sallis et al., 1993) and have increased risk of obesity (Ogden et al., 2012). Both physical and social environmental factors influence young children's physical activity (Dolinsky et al., 2011; Dowda et al., 2011). Yet, little is known about where young children are likely to be active.

The ability to simultaneously and objectively capture physical activity and location data on young HispanicAmerican children may provide more precise findings

\footnotetext{
Corresponding author:

Teresia M. O'Connor

USDA/ARS Children's Nutrition Research Center

Academic General Pediatrics Baylor College of Medicine

1100 Bates Street, Houston, TX 77030, USA

Tel. +1 713798 6782; Fax +1 7137986782

E-mail: teresiao@bcm.edu
}

to inform better environmental interventions and policy changes to promote physical activity. Although the combined objective assessment of location and physical activity via global positioning system (GPS) data loggers and accelerometers has been demonstrated feasible and useful among adults and older children (Duncan et al., 2009; Wheeler et al., 2010; Krenn et al., 2011; Almanza et al., 2012), little is known about the feasibility of simultaneously using these monitors with preschool-aged children. Only two studies assessed the feasibility and validity of using GPS tracking devices to identify the location of preschool children (Elgethun et al., 2003, 2007). Neither study simultaneously assessed the child's physical activity. One study assessed indoor versus outdoor activity in preschool children using GPS and accelerometer, but did so only in the school context (Tandon et al., 2013).

The aims of this study were to: (i) assess the feasibility to recruit participants and simultaneously obtain location and activity data on Hispanic preschool children in real life settings; (ii) assess the agreement of child location and travel mode as measured by GPS data loggers with parent-reported diaries as the valid- 
ity criterion; and (iii) explore the utility of GPS monitors and accelerometers to identify locations where Hispanic-American preschool children were more physically active.

\section{Methods}

\section{Participants}

Fifteen Hispanic-American children, 3-5 years old, residing in Houston, Texas were recruited via fliers posted in the community and at the Texas Medical Center, and from the Children's Nutrition Research Center's volunteer list. The average age of the children was 4.7 (SD 0.8 ) years; $60 \%$ were boys; and $53 \%$ of the families reported household incomes of less than US $\$ 50,000 /$ year. Data were collected in JanuaryFebruary 2011.

\section{Instruments and measures}

Monitors: Children simultaneously wore QStarz BT100X GPS data loggers (Qstarz International Co., Ltd; Taiwan) and Actigraph GT3X accelerometers (Actigraph, Pensacola, USA) whose clocks were synchronised to the Universal Time Clock, for a 24- to 36-hour period on a day when the parent and the child were to spend the whole day together. Physical activity data were collected at $30 \mathrm{sec}$ epochs and GPS data were collected at 5 or $30 \mathrm{sec}$ epochs to test monitor memory abilities. The monitors were worn on two separate elastic belts each fitted on the child's hips, after noting that wearing both monitors on one belt (as recommended for adults; see Kerr et al., 2011) became too heavy for younger children. Children were instructed to wear the monitors at all times during the study period except while swimming or bathing to prevent the monitors from getting wet. Parents were instructed that they could remove the GPS monitor from their child whenever they did not want the study monitors to track their child's location.

Location diaries: Parents completed a location and travel behaviour diary for 12 hours (08:00 to 20:00 hours) on the same day the monitors were worn, at 5 min intervals. The diary specified the child's current location by micro-environmental categories (home, other residential house, store, restaurant, church, community centre, park, other); whether the child was indoors or outdoors; and if the child was travelling, along with mode of travel (car, bus, walking or stroller). Each variable in the diary was indicated by a check box. Parent reported location diaries have previously been used to assess preschool children's locations for environmental exposures (Kawahara et al., 2012) and as criterion validity with early GPS devices (Elgethun et al., 2007). In addition, the parent documented if the child removed one or both monitors during the study period. At the end of the study period, research staff members reviewed the diaries with the families and clarified any entries that were unclear to help improve the accuracy of the data. Diaries were returned with greater than $95 \%$ of the time intervals completed for the 12-hour study period.

Upon return of the diaries and monitors with complete data (at least 480 min of activity and GPS data), the family received US\$30 compensation. To be eligible for compensation, participants with fewer than 8 hours of valid accelerometer data were asked to wear the monitors a second time. The Baylor College of Medicine Institutional Review Board approved the protocol. Parents provided written informed consent for their child to participate.

\section{Data processing and analyses}

There are no agreed upon data reduction algorithms for accelerometer data among preschool-aged children (Cliff et al., 2009). Previous studies in children and adults have elected to remove $10,20,30$ or $60 \mathrm{~min}$ of consecutive zeros as non-wear time (Masse et al., 2005; Cliff et al., 2009), a decision which may influence the number of valid days included for each participant. In this study, accelerometer data were considered completed if there were $480 \mathrm{~min}$ of data after excluding consecutive zero activity for $30 \mathrm{~min}$ or more (i.e. non-wear time). Child physical activity was reported as activity counts per $30 \mathrm{sec}$ epoch. GPS data were matched to valid accelerometer data.

Data from both monitors were simultaneously processed using the Personal Activity Location Measurement System, version 1.0.6 (PALMS: https://palms.ucsd.edu:8443/PALMS/) (Patrick et al., 2008; Kerr et al., 2011; Demchak et al., 2012). PALMS is an encrypted web application that simultaneously processes time-stamped accelerometer and GPS data to clean, filter and detect locations and travel mode based on study specified settings and established algorithms. Locations were identified in PALMS for GPS coordinates with a $100 \mathrm{~m}$ buffer where the participant spent at least $3 \mathrm{~min}$. The locations identified in PALMS were viewed using Google Earth and coded as whether they matched the location recorded in the diary. The time spent in each 
location as assessed via PALMS output and the diary was compared. PALMS also identified the time a participant was taking a trip between locations. Trip parameters were set at travel of $\geq 30 \mathrm{~m} / \mathrm{min}$, with a minimum of $100 \mathrm{~m}$ for a duration $\geq 180 \mathrm{sec}$. Mode of travel was differentiate vehicle versus non-vehicular (from now on referred to as walking) with the vehicle speed cut-off as $\geq 40 \mathrm{~km} / \mathrm{h}$ and walking as $2-40 \mathrm{~km} / \mathrm{h}$ as per PALMS default settings established for adults (Kerr et al., 2012).

For each participant, observation-by-observation correspondence between GPS- and diary-assessed locations and travel mode were estimated with Kappa statistics and percent agreement. Whole-sample point estimates and relative standard deviations of Kappa statistics and percent agreement were computed. Between-method differences in total min spent at a location or in travel, aggregated at the person level, were estimated and tested for statistical significance using paired $t$-tests and Wilcoxon signed-ranks tests. Between-method associations of total min spent at a location or in travel, aggregated at the person level, were estimated using Kendall's tau correlation coefficient. The association of observation-by-observation of children's location with their objectively measured physical activity were explored using multilevel models. Children's physical activity (outcome variable) was quantified as the accelerometer-based activity counts per min. All models were adjusted for dependency in observations (repeated measures on the same subjects).

\section{Results}

Parents were receptive to having their child wear monitors. All children were able to wear both monitors simultaneously and had valid GPS data. There were no apparent monitor removals by parents because they did not want their child's location tracked. Eighty percent $(n=12)$ of children had valid physical activity data with a mean of 10.0 hours (SD 2.7), including one re-wear. These data were used in subsequent analyses. Invalid accelerometer data were primarily due to accelerometer initialization problems, rather than non-wear.

The average correspondence between GPS- and diary-based estimates of location types ranged from $91.9 \%$ to $99.9 \%$ agreement with moderate to good Kappa statistics, ranging from 0.49 to 0.99 (moderate to almost perfect) (Table 1). The between-method (GPS monitors and parent reported diaries) correlation of estimated participant-aggregated min spent on vehicle-based trips was strong (Spearman correlation $0.65, \mathrm{P}<0.01)$. However, those related to walking trips and total trips were weak and not statistically significant (Spearman correlation 0.28 and 0.37 respectively, $\mathrm{P}>0.05$ ) (Fig. 1). Of note, only two children had walking trips documented during the study period. The average observation-by-observation correspondence between GPS- and diary-based estimates of travelmode ranged from $87.4 \%$ to $97.3 \%$ agreement, and Kappa statistics ranged from 0.31 to 0.53 (fair to moderate agreement). Children's mean physical activity counts per min varied by location (Table 2 ). The

Table 1. Average time spent in specific locations by method of assessment, correlations and average observation-by-observation correspondence between diary-based and GPS (processed by PALMS) measures of locations.

\begin{tabular}{|c|c|c|c|c|c|c|c|c|c|c|}
\hline \multicolumn{7}{|c|}{$\begin{array}{l}\text { Minutes spent in location and } \\
\text { standard deviation }(\mathrm{SD})\end{array}$} & \multicolumn{4}{|c|}{$\begin{array}{l}\text { Observation-by-observation } \\
\text { correspondence }\end{array}$} \\
\hline \multirow{2}{*}{ Location } & \multicolumn{2}{|c|}{ GPS } & \multicolumn{2}{|c|}{ Diary } & \multirow{2}{*}{$\begin{array}{l}\text { Kendall's } \\
\text { tau }\end{array}$} & \multirow{2}{*}{ P-value } & \multicolumn{2}{|c|}{ Agreement } & \multicolumn{2}{|c|}{ Kappa } \\
\hline & $\min$ & $\mathrm{SD}$ & $\min$ & SD & & & $\%$ & SD & Value & SD \\
\hline Child's home & 464.5 & 182.9 & 444.0 & 160.9 & 0.87 & $<0.001$ & 92.0 & 6.9 & 0.79 & 0.18 \\
\hline Other home & 54.7 & 112.8 & 65.1 & 112.4 & 0.63 & 0.021 & 91.9 & 20.0 & 0.49 & 0.50 \\
\hline Store & 55.3 & 72.4 & 55.0 & 76.1 & 0.96 & $<0.001$ & 97.1 & 4.3 & 0.78 & 0.21 \\
\hline Restaurant & 13.5 & 32.2 & 12.1 & 29.2 & 1.00 & 0.001 & 99.5 & 1.2 & 0.84 & 0.04 \\
\hline Church* & 2.3 & 7.8 & 6.3 & 21.7 & 1.00 & 0.003 & 99.2 & 2.3 & 0.45 & $\mathrm{NA}$ \\
\hline Community centre & 21.8 & 57.5 & 21.5 & 56.5 & 1.00 & 0.001 & 99.9 & 0.2 & 0.99 & 0.10 \\
\hline Park & 5.3 & 12.4 & 5.4 & 12.7 & 1.00 & 0.001 & 99.7 & 1.0 & 0.78 & 0.22 \\
\hline Other location & 17.5 & 29.4 & 18.4 & 33.5 & 0.79 & 0.004 & 99.3 & 1.3 & 0.78 & 0.17 \\
\hline
\end{tabular}

*Only one participant visited a church during the monitoring period. Hence, only one kappa statistic was computed.

Note: values represent means and standard deviations of statistics obtained on 12 participants. 


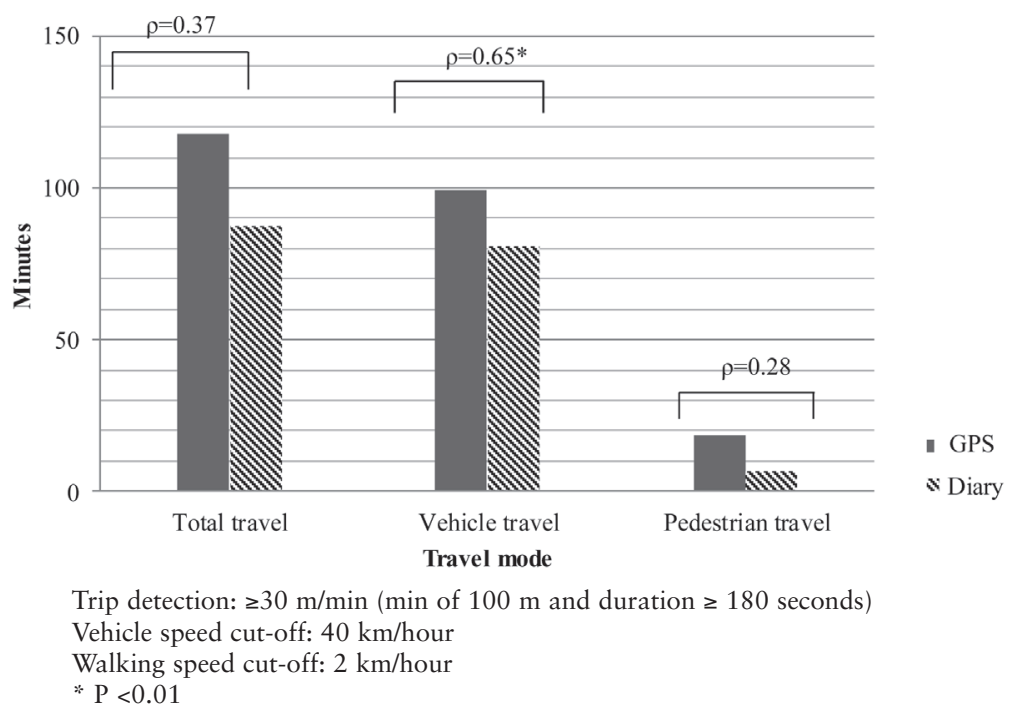

Fig. 1. Time spent in travel mode per diary-based and GPS (processed by PALMS) measures of trips with Spearman correlations ( $\rho)$.

association of activity to location was in the same direction whether assessed by parent diary or GPS monitor. Children were significantly more active in parks and less active in restaurants compared with at home, when estimating locations by diaries as well as GPS monitors (Table 2).

\section{Discussion}

This is one of the first studies to demonstrate that simultaneous objective assessment of location and physical activity is possible for preschool-aged children providing rich contextual data on where children

Table 2. Predictors of activity counts (adjusted for person-level clustering effects).

\begin{tabular}{|c|c|c|c|}
\hline Predictors & $\exp (b)$ & $95 \%$ Confidence Interval & P-value \\
\hline \multicolumn{4}{|c|}{ Trip (GPS) reference: no trip } \\
\hline Vehicle & 0.57 & $0.41, \quad 0.84$ & 0.003 \\
\hline Walking & 0.85 & $0.38, \quad 1.87$ & 0.685 \\
\hline \multicolumn{4}{|c|}{ Trip (diary) reference: no trip } \\
\hline Vehicle & 0.48 & $0.35, \quad 0.66$ & $<0.001$ \\
\hline Walking & 1.18 & $0.70, \quad 1.60$ & 0.284 \\
\hline \multicolumn{4}{|c|}{ Location (diary) reference: child's home } \\
\hline Other home & 1.36 & $0.51, \quad 0.84$ & 0.539 \\
\hline Store & 1.15 & $0.71,1.86$ & 0.570 \\
\hline Restaurant & 0.35 & $0.22, \quad 0.56$ & $<0.001$ \\
\hline Church & 0.91 & $0.61,1.37$ & 0.662 \\
\hline Community centre & 0.69 & $0.39,1.22$ & 0.198 \\
\hline Park & 1.77 & $1.19,2.64$ & 0.005 \\
\hline Other locations & 0.67 & $0.37, \quad 1.21$ & 0.185 \\
\hline \multicolumn{4}{|c|}{ Location (GPS) reference: child's home } \\
\hline Other home & 3.24 & $0.95, \quad 11.03$ & 0.060 \\
\hline Store & 1.22 & $0.72, \quad 2.08$ & 0.458 \\
\hline Restaurant & 0.50 & $0.24, \quad 1.00$ & 0.050 \\
\hline Church & 1.74 & $1.22, \quad 2.47$ & 0.002 \\
\hline Community center & 0.76 & $0.43,1.33$ & 0.331 \\
\hline Park & 2.23 & $1.50,3.31$ & $<0.001$ \\
\hline Other locations & 0.72 & $0.35,1.49$ & 0.382 \\
\hline
\end{tabular}

Note: generalized linear mixed models with negative binomial variance and logarithmic link function. Reference: reference category; $\operatorname{Exp}(b)=$ antilogarithm of regression coefficient; $95 \% \mathrm{CI}=$ antilogarithms of $95 \% \mathrm{CI}$; Each predictor estimated in separate model. $\operatorname{Exp}(b)$ : interpretation $\geq$ if $\exp (b)>1.00$, predictor category associated with $(\exp (b)-1) * 100 \%$ higher average activity counts than the reference category; if $\exp (b)<1.00$, predictor category associated with $(1-\exp (b)) * 100 \%$ lower average activity counts than the reference category. All models adjusted for dependency in observations (repeated measures on the same subjects). 
are more active. The accelerometer and GPS data loggers provided automatic data collection that could be synchronised with little-to-no burden on families. High between-method correspondence was found for location compared to parent report via a time-intensive activity diary that is not feasible for use for long periods of time. Moderate correspondence was observed for mode of travel. Low correspondence between methods of assessing walking was likely due in part to very few children participating in any walking trips during the study period and may be improved by refining the pedestrian trip cut-offs specifically for preschool children in future validation studies. Simultaneous objective assessment of location and physical activity among high school and middle school students has demonstrated that physical activity is associated with mode of transport to school (Cooper et al., 2010) and proximity of physical activity to home and school (Maddison et al., 2011). The present results suggest that these technologies are also useful in assessing environmental contexts that promote physical activity for younger children.

Simultaneous objective assessment of the child's location and physical activity demonstrated that the location of the child was associated with how active she was, even in these exploratory analyses. Greater counts per min of activity were accumulated while children were in parks compared to any other location, regardless of how the location was assessed (diary or GPS). Similarly, others have found that older children's physical activity increased by the amount of exposure to green spaces as assessed by GPS (Almanza et al., 2012). In the present study, the association between location and child physical activity were in the same direction whether the location was assessed by parent diary or GPS monitor. However, the strength of the association varied by the method of assessing the child's location, suggesting that more objective measurements of location may improve accurate assessment of effect sizes (Kerr et al., 2011).

This small feasibility study had several limitations. The study duration was for one day to minimise the response burden for parents to complete the activity and location diaries at $5 \mathrm{~min}$ intervals, which is shorter than typically recommended to accurately assess children's habitual physical activity (Trost et al., 2000). The location diary, which was the comparison criterion for GPS data to identify location and travel mode has inherent reporting biases. Direct observational methods would have likely provided more objective and valid criterion against which to compare the GPS data, but they are intrusive and not feasible for a full day assessment with a family in a natural home and community setting. There were no qualitative data collected from the parents or children after the assessment period, which could have gauged any burden on the child of wearing two monitors or sense of intrusion into privacy for tracking the child's location. Future studies should explore this in detail.

A strength of the study was using standard methods for simultaneously processing the activity and GPS data using software available to others at PALMS: https://palms.ucsd.edu:8443/PALMS/ (Patrick et al., 2008). Future work needs to validate the PALMS algorithms for preschool-aged children to better characterise their physical activity and correctly identify their mode of transportation from one location to another.

The simultaneous use of accelerometers and GPS monitors to assess young children's physical activity and location will allow scientists to investigate the complex effect of the physical environment on preschool children's physical activity. These methods should be considered for use in descriptive and intervention studies to better identify the role that the environment and location context have on children's activity. Such studies could inform policy decisions about the location, accessibility and maintenance of safe outdoor spaces for children's activity.

\section{Acknowledgements}

We would like to thank Drs. Kevin Patrick and his research group at the University of California, San Diego for providing us access and very helpful assistance in using the PALMS software system to simultaneously process the GPS and accelerometer data. This work is a publication of the US Department of Agriculture (USDA/ARS) Children's Nutrition Research Center, Department of Pediatrics, BCM funded in part by the USDA/ARS (Cooperative Agreement 6250-51000-053-20S). The content of this publication does not necessarily reflect the views or policies of the USDA, nor does mention of organizations imply endorsement from the US government. This project was funded by a R21 grant from NIH-NICHD (5R21HD060925, PI: O’Connor). JAM was supported, in part, by a career development award (K07CA131178). The analysis in this paper was supported in part through collaboration with the PALMS Project (UCSD-PalmsProject.wikispaces.com) at the University of California, San Diego, funded by NIH/NCI Grant 1 U01 CA130771.

\section{References}

Almanza E, Jerrett M, Dunton G, Seto E, Pentz MA, 2012. A study of community design, greeness, and physical activity in children using satellite, GPS, and accelerometer data. Health Place 18, 46-54. 
Cliff D, Reilly J, Okely A, 2009. Methodological considerations in using accelerometers to assess habitual physical activity in children aged 0-5 years. J Sci Med Sport 12, 557-567.

Cooper A, Page A, Wheeler B, Griew W, Davis L, Hillsdon M, 2010. Mapping the walk to school using accelerometry combined with a global positioning system. Am J Prev Med 38, 178-183.

Demchak B, Kerr J, Raab F, Patrick K, Krüger IH, 2012. PALMS: a modern coevolution of community and computing using policy driven development [Abstract]. 45th Hawaii International Conference on System Sciences (HICSS), Maui, Hawaii.

Dolinsky D, Brouwer R, Evenson K, Siega-Riz A, Østbye T, 2011. Correlates of sedentary time and physical activity among preschool-aged children. Prev Chronic Dis 8, A131.

Dowda M, Pfeiffer K, Brown W, Mitchell J, Byun W, Pate R, 2011. Parental and environmental correlates of physical activity of children attending preschool. Arch Pediat Adol Med 165, 939-944.

Duncan S, Badland H, Schofield G, 2009. Combining GPS with heart rate monitoring to measure physical activity in children: a feasibility study. J Sci Med Sport 12, 583-585.

Elgethun K, Fenske R, Yost M, Palcisko G, 2003. Time-location analysis for exposure assessment studies of children using a novel global positioning system instrument. Environ Health Persp 111, 115-122.

Elgethun K, Yost M, Fitzpatrick C, Nyerges T, Fenske R, 2007. Comparison of global positioning systems (GPS) tracking and parent-report diaries to characterize children's time-location patterns. J Expo Sci Env Epid 17, 196-206.

Kawahara J, Tanaka S, Tanaka C, Aoki Y, Yonemoto J, 2012. Daily inhalation rate and time-activity/location pattern in Japanese preschool children. Risk Anal 32, 1595-1604.

Kerr J, Duncan S, Schipperjin J, 2011. Using global positioning systems in health research: a practical approach to data collection and processing. Am J Prev Med 41, 532-540.
Kerr J, Norman G, Godbole S, Raab F, Demchak B, Patrick K, 2012. Validating GPS data with the PALMS system to detect different active transportation modes. Med Sci Sport Exer 44, S25-S29.

Krenn P, Mag D, Titze S, Oja P, Jones A, Ogilvie D, 2011. Use of global positioning systems to study physical activity and the environment. Am J Prev Med 41, 508-515.

Maddison R, Jiang Y, Hoorn S, Exeter D, Mhurchu C, Dorey E, 2011. Describing patterns of physical activity in adolescents using global positioning systems and accelerometry. Pediatr Exerc Sci 22, 392-407.

Masse LC, Fuemmeler BF, Anderson CB, Matthews CE, Trost SG, Catellier DJ, 2005. Accelerometer data reduction: a comparison of four reduction algorithms on select outcome variables. Med Sci Sport Exer 37, S544-S554.

Ogden CL, Carroll MD, Kit BK, Flegal KM, 2012. Prevalence of obesity and trends in body mass index among US children and adolescents, 1999-2010. JAMA 307, 483-490.

Patrick K, Kerr J, Norman G, Ryan S, Sallis J, Krueger I, 2008. Geospatial measurement and analysis of physical activity: physical activity location measurement system (PALMS). Epidemiology. ISEE 2008 Conference Abstracts Supplement 19, S63.

Sallis J, Nader P, Broyles S, 1993. Correlates of physical activity at home in Mexican-American and Anglo-American preschool children. Health Psychol 12, 390-398.

Tandon PS, Saelens BE, Zhou C, Kerr J, Christakis DA, 2013. Indoor versus outdoor time in preschoolers at child care. Am J Prev Med 44, 85-88.

Trost S, Pate R, Freedson P, Sallis J, Taylor W, 2000. Using objective physical activity measures with youth: how many days are needed? Med Sci Sport Exer 32, 426-431.

Wheeler B, Cooper, A, Page A, Jago R, 2010. Greenspace and children's physical activity: a GPS/GIS analysis of the PEACH project. Prev Med 51, 148-152. 